\title{
Performance Analysis of AntNet-LA Protocol for Ad-hoc Networks based on Disaster Area Mobility Model
}

\author{
Vaibhav Godbole \\ Fr. Conceicao Rodrigues College Of Engineering, Mumbai-400050, India \\ Correspondence: Vaibhav Godbole, vai.godbole@gmail.com \\ Manuscript communication: received 4 March 2013, accepted 9 June 2013
}

\begin{abstract}
Availability of cheap positioning instruments like GPS receivers makes it possible for routing algorithms to use the position of nodes in an ad hoc mobile network. Regular position based routing algorithms fail to find a route from a source to a destination in some cases when the network contains nodes with irregular transmission ranges or they find a route that is much longer than the shortest path. On the other hand routing algorithms based on Ant Colony Optimization (ACO) find routing paths that are close to the shortest paths even if the nodes in the network have different transmission ranges. The drawback of these algorithms is the large number of messages that needs to be sent or the long delay before the routes are established. In this paper, we propose a novel protocol AntNet-LA which combines the idea of ACO with information about position of all nodes. In this technique the distance between the nodes is considered to transmit the packets, hence overcomes the drawbacks of AntNet algorithm which considers only cumulative probability for packet transmission. We compare performance of AntNet-LA with AntNet, Ad-hoc On Demand Distance Vector (AODV), Ad-hoc On Demand Multipath Distance Vector (AOMDV), Dynamic Source Routing (DSR) and Destination-Sequenced Distance-Vector Routing (DSDV) protocols. We also compare performance of AntNet-LA with distance-aware protocols such as Location Aided Routing (LAR), Geographical AODV GeoAODV and Position Based ANT colony optimization (PBANT).
\end{abstract}

Keywords- Ant colony optimization, modified AntNet, AntNet-LA, location aware protocol

\section{INTRODUCTION}

Ant algorithms are an iterative, probabilistic metaheuristic for finding solutions to combinatorial optimization problems. They are based on the foraging mechanism employed by real ants attempting to find a short path from their nest to a food source. While foraging, the ants communicate indirectly via pheromone, which they use to mark their respective paths and which attracts other ants. In the ant algorithm, artificial ants use virtual pheromone to update their path through the decision graph, i.e. the path that reflects which alternative an ant chooses at certain points. Ants of the later iterations use the pheromone marks of previous good ants as a means of orientation when constructing their own solutions, which ultimately result in focusing the ants on promising parts of the search space. In sometimes, a problem might be dynamic in nature, changing over time and requiring the algorithm to keep track of the occurring modifications and continually re-optimize in order to be able to present a valid, good solution at all times. Ant algorithms have a number of attractive features, including adaptation, robustness and decentralized nature, which are well suited for routing in modern communication networks such as Mobile Ad hoc Networks (MANETs).

In 1998 AntNet algorithm was proposed by [1], which is based on Ant Colony Optimization (ACO) with mobile software agents. This algorithm establishes a path from source to destination in purely random fashion.
The learning structure of AntNet consists of colonies of learning automata. In 2010, Pooia Lalbakhsh [2] have proposed to replace the previously defined automata structure with the non-linear one, modifies the reinforcement process without imposing overhead into the system. In this paper, in order to select the appropriate non-linear functions, the convergence rates are mathematically analyzed and the functions with better rates are replaced at the core of the system's learning cycle. This modified version of AntNet improves the system adaptability in the presence of undesirable events.

The adaptive routing algorithm we propose in this paper is distributed and mobile multi-agent systems well matching the characteristics of the general routing problem. The design of our algorithms has been inspired by previous works on ant colonies and, more generally, by the notion of stigmergy that is, the indirect communication taking place among individuals through local, persistent (or slowly changing) modifications induced in their environment. Real ants have been shown to be able to find shortest paths using a stochastic decision policy based only on local information represented by the pheromone trail deposited by other ants. Algorithms that take inspiration from ant's behavior in finding shortest paths have recently been successfully applied to several discrete optimization problems. In ant colony optimization each one of a set of con-current artificial ants makes use of a stochastic local search strategy to build a solution to the combinatorial problem under consideration. The whole set of 
Table I

COMPARISON BETWEen Ad HOC Network ANd ANTS

\begin{tabular}{|l|l|l|}
\hline Parameters & $\begin{array}{l}\text { Ad-hoc Net- } \\
\text { works }\end{array}$ & Ants \\
\hline Overhead & More & Less \\
\hline $\begin{array}{l}\text { Packet Delivery } \\
\text { ratio }\end{array}$ & Less & More \\
\hline $\begin{array}{l}\text { Route Discovery } \\
\text { Procedure }\end{array}$ & $\begin{array}{l}\text { Route Re- } \\
\text { quest/Reply } \\
\text { message are } \\
\text { used }\end{array}$ & $\begin{array}{l}\text { To provide } \\
\text { definite } \\
\text { shortest path }\end{array}$ \\
\hline Motive & $\begin{array}{l}\text { Find shortest } \\
\text { path for rout- } \\
\text { ing }\end{array}$ & $\begin{array}{l}\text { To provide } \\
\text { definite } \\
\text { shortest path }\end{array}$ \\
\hline Path Discovered & $\begin{array}{l}\text { Single path, } \\
\text { partially } \\
\text { multipath }\end{array}$ & Multipath \\
\hline
\end{tabular}

ants collectively search for high quality solutions by a cooperative effort mediated by indirect communication of information on the problem structure they collect while building solutions.

While comparing Ad-hoc networks and ants in Table I we come to conclusion that though they have similarities like same physical structure, self-configuration and self-organization but still distinguished from each other in the route foundation, overhead, motive, routing table information. Ant based routing algorithms exhibit a number of desirable properties for ad hoc networks. The foraging behavior of ants, bees and the hill building behavior of termites has inspired researchers in developing efficient routing algorithm for ad hoc networks. The remainder of this paper is organized as follows. In Section 2 we present related work, in Section 3 we present drawbacks of AntNet algorithm, in Section 4 we present our proposed AntNet-LA algorithm. Subsequently in Section 5 we present the working of AntNetLA algorithm with an example, in Section 6 we present deployment of AntNet-LA, in Section 7 we present performance evaluation parameters, in Section 8 we present simulation results of AntNet-LA, in Section 9 we present other possible applications of AntNet-LA and finally a conclusion is given in Section 10 .

\section{Related Work}

In this section we present location-aware protocols and ACO algorithms, which are applied to ad-hoc networks. Location-aware protocols uses information of co-ordinates to transmit the packets [3] whereas ACO algorithms are inspired by behavior of ants in nature.

\subsection{Location Aided Routing Protocols}

In this section we review some location-aware protocols for ad-hoc networks.

A number of location-aware protocols were proposed. Location Aided Routing (LAR) [4] is one of the most popular mechanisms for reducing control message overhead in Ad-hoc On Demand Distance Vector
AODV [5]. LAR assumes that the nodes in the network know their own location and can obtain the last known position of the destination node.Location information used in the LAR protocol may be provided by the Global Positioning System (GPS). With the availability of GPS, it is possible for a mobile host to know its physical location. In reality, position information provided by GPS includes some amount of error, which is the difference between GPS-calculated coordinates and the real coordinates. LAR modifies the route discovery process so that only the nodes which belong to the search area would rebroadcast RREQ messages. Specifically, when an RREQ packet arrives at an intermediate node, the node first determines whether it belongs to the search area defined by the incoming RREQ message. If the node is part of that search area then the RREQ packet is re-broadcasted, otherwise the packet is discarded.

Geographical AODV (GeoAODV) [6] is another location based protocol which takes advantage of GPS and assumes that each communicating device has GPS access and knows its location. Using GPS coordinates GeoAODV limits the route discovery process to the search region that is likely to contain the path to destination. Only nodes inside of the search region are allowed to rebroadcast RREQ messages during the route discovery process. This is a modified version of LAR variation called cone-shaped request zone adaptation [4]. However, unlike LAR, our proposed approach does not make any assumptions regarding the speed and direction of the moving nodes. Instead, our approach relies only on GPS coordinates to determine the search region that is likely to contain the path to destination. The main contributions of our work are providing evaluation and detailed description of the GeoAODV approach as well as introducing a new protocol for maintaining and distributing GPS coordinates in the network.

Position Based ANT colony optimization (PBANT) [7] is a combination of both Position Based Routing Algorithms and Ant Colony Based Routing Algorithms. PBANT uses location information of the nodes to improve its efficiency and reduce the algorithm overhead. As PBANT is reactive, a route is searched for only when there is a collection of data packets that are to be sent from a source node $S$ to a destination node $\mathrm{D}$ using the concept of zoning. To form zones, a line is drawn between source and destination node. Then the angle between source mode and neighboring node is used to decide the zones. PBANT assumes that each node maintains a table of values of the pheromone trails assigned to its outgoing links for different destinations. Whenever a node receives a packet for a specific destination, it will check its table to see if there is at least one pheromone trail for that destination. If such pheromone trail exists, it will be used for making a stochastic decision to choose the next hop. If it doesn't exist, the pheromone initialization process begins and assigns pheromone trails to all the outgoing links. The amount of the deposited pheromone on each link depends on the zone of the corresponding neighbor. The pheromone 


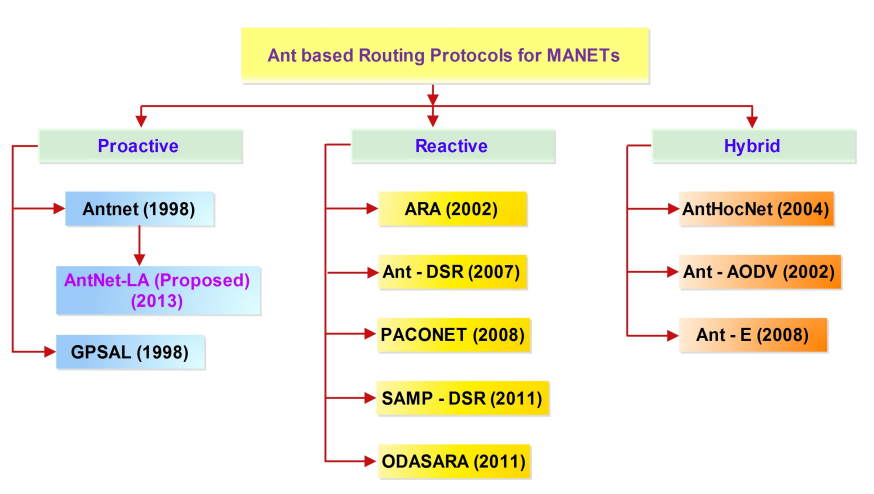

Figure 1. A few ACO based protocols for MANETs.

Table II

Ants based Routing Protocols for Ad-hoc Networks

\begin{tabular}{|c|c|c|c|c|}
\hline Algorithms & Year & Authors & $\begin{array}{l}\text { Routing } \\
\text { type }\end{array}$ & $\begin{array}{l}\text { Path } \\
\text { type }\end{array}$ \\
\hline $\mathrm{ABC}$ & 1997 & Schoonerwood & Proactive & Single \\
\hline AntNet & 1998 & $\begin{array}{l}\text { Di Caro et } \\
\text { al. [1] }\end{array}$ & Proactive & Single \\
\hline ARA & 2002 & Gunes et al. & Reactive & Multipath \\
\hline AntHocNet & 2004 & $\begin{array}{l}\text { Di Caro et } \\
\text { al. [11] }\end{array}$ & Hybrid & Single \\
\hline ACS & 2007 & $\begin{array}{l}\text { A. Mora et } \\
\text { al. [12] }\end{array}$ & Hybrid & Multipath \\
\hline PACONET & 2008 & Osagie et al. & Reactive & Single \\
\hline
\end{tabular}

trails for a specific destination will be deleted from the pheromone trail table of a node if the node doesn't receive any packet pointing to that destination for more than a specific time which is defined to be in the order of seconds. The drawback of PBANT is that if the network graph is not fully connected, the source and destination may reside in different partitions which mean that there is no route to the destination. This means that in this case the packet can not reach to destination at all.

\subsection{Ant Colony Optimization Algorithms}

In the nature, ants lay pheromone and so they produce pheromone trails between the nest and a food source. On a computer, the pheromone has been replaced by artificial stigmergy, the probabilities in the routing tables. To compute and update the probabilities, intelligent agents are introduced to replace the ants. There exist two kinds of agents, the forward agents and the backward agents. All forward and backward agents have the same structure. The agents move inside the network by hopping at every time step from a node to the next node along the existing links. The agents communicate with each other in an indirect way by concurrently reading and writing the routing tables on their way $[8,9]$. There exist a number of swarm intelligence based protocols but the most important are ant based protocols [10]. Table II and Figure 1 shows a few of the ant based routing algorithms.

Table III

Selection Probability at Node 2

\begin{tabular}{|l|l|l|l|l|l|}
\hline Next hop & 1 & 4 & 3 & 6 & 8 \\
\hline Probability of routing & 0.12 & 0.10 & 0.18 & 0.15 & 0.45 \\
\hline Cumulative probability & 0.12 & 0.22 & 0.40 & 0.55 & 1.0 \\
\hline
\end{tabular}
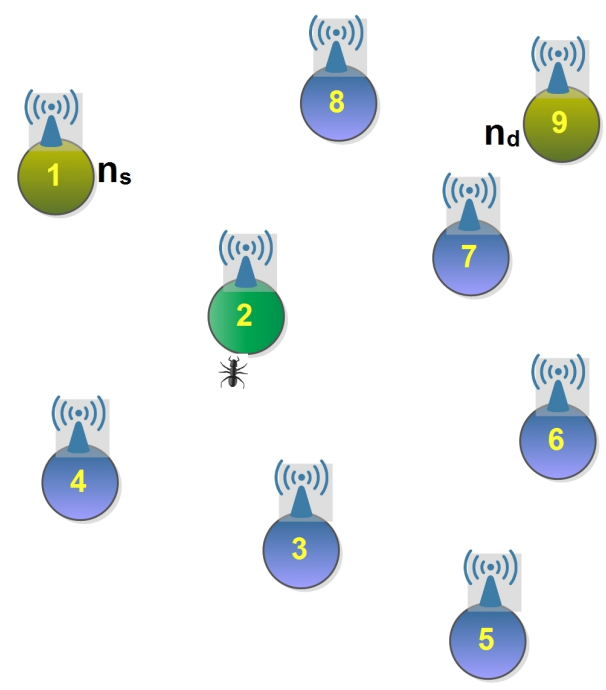

Figure 2. Example of a network with 9 nodes

\section{Drawbacks of AntNet Algorithm}

Consider an example of a network which consists of 9 nodes as shown in Figure 3. It is required to transmit the packet from source node $n_{S}(1)$ to destination node $n_{d}$ (2). At present the packet is at node 2. Table III shows the next hop selection process at node 2 . The AntNet algorithm will forward the packet based on random number generated. If the generated random number is 0.1 , then node 1 will be selected as a next hop. This may cause looping problem. Then the packet will be forwarded to node 1 and duplicate cycle will be deleted from the memory of ant. If the generated random number is 0.5 then node 6 will be selected as next hop. Hence in AntNet algorithm, the decision to select next hop will depend upon the generated random number and cumulative probability. The AntNet algorithm is having following drawbacks:

1) As the AntNet algorithm forwards the packets to the next hop depending upon the probability values only [13], the path followed by the ants may not be optimum every time.

2) The distance between the source node and the next node is not considered by AntNet algorithm, even though the traveling time of ants is considered to generate the probability values.

Hence the end to end delay would very high. The high end to end delay is not tolerable in some applications, which uses datagram packets.

We have modified AntNet algorithm in such a way that if the random number generated by AntNet algorithm is less than pre-defined re-enforcement factor $r$, and if it is small enough, instead of choosing the route destination as the AntNet algorithm would, the algorithm will compare the node's relative orientation with 
respect to the neighbouring nodes and the destination node for the packet, and send the packet to the neighbouring node closest to the destination node. Then the routing decision changes the node's pheromone table as normal AntNet routing would. In other cases where random number generated by AntNet algorithm is larger than the pre-defined value of $r$, then the packet is forwarded as per normal AntNet routing algorithm based on the pheromone levels in the routing table.

For comparing distance between source node, neighboring node and destination node, we propose a technique to find the distance between two nodes. This algorithm is called as AntNet-LA.

\section{AntNet-LA Algorithm}

\subsection{Forward Ants}

Each node s periodically sends a forward ant (forward agent) to the neighboring agent chosen by the AntNet-LA algorithm. Then this neighboring node again compares the re-enforcement factor $r$ with the predefined value. This process continues till the present value of $r$ becomes greater than the predefined value.The task of the forward ant is to discover a feasible, low-cost path to the destination and to gather useful information on its trip. Every ant will represent a package sent from $\mathrm{s}$ to $\mathrm{d}$ through the network and each forward ant packet contains the following fields; id number of source node, id number of destination node and stack memory.

At every visited node $k$ on the way to the destination node, a forward ant does the following:

- The forward ant checks its stack memory whether node $\mathrm{k}$ has already been visited before or not. If it has been visited, there will be a cycle in the ant's path and this cycle will be deleted from the memory. The cycle's nodes are popped from the ant's stack and all the memory about them is destroyed.

- In the first iteration of our algorithm, all the links from a node $k$ to its neighbor nodes have equal probabilities, so the forward ant goes randomly to any one of its neighbor nodes.

- In the successive iterations, the selection of the next hop is done by comparing $r$ values with predefined one.

- If node $k$ is the destination node then the forward ant transforms to a backward ant.

The individuals (nodes of each routing table) are mapped to contiguous segments of a line, such that each node's segment is equal in size to its fitness (probability of routing). A random number between 0 and 1 is generated for every link to a neighbor node according to the magnitude of the probabilities and the node whose segment spans the random number is selected.

Now, all forward ants arrives at their destination node. Each ant has a stack of the virtual elapsed time for each ant trip. The stack of the forward ant is a dynamically growing data structure that contains the id number of the nodes that the forward ant has traversed as well as the elapsed time between its starting from $s$ to its arriving to node $k$.

\subsection{Backward Ants}

The backward ant (backward agent) inherits the memory from the forward ant. The task of the backward ant is to go back to the source node s along the same path as the forward agent but in the opposite direction and to update the routing tables on this path. Once the path is updated the backward ant reaches the source node and dies

- The backward agent updates the routing table of node $k$ by using the travel times stored in its memory.

- If node $k$ is not the source node then the backward agent uses its memory to determine the next link of the path back to the source node. The backward agent goes to the next node along that link.

- If node $k$ is the source node then the backward agent is killed.

\subsection{Updating Routing Tables}

Updating in routing tables at each node will be done by backward ants using ants trip times. As shown in Figure 3 at every visited node $k$ on the way back to the source node, a backward ant updates some of the probabilities in the routing table of node $\mathrm{k}$ by using the travel information in its memory which was collected by the forward ant. The backward ant retraces the

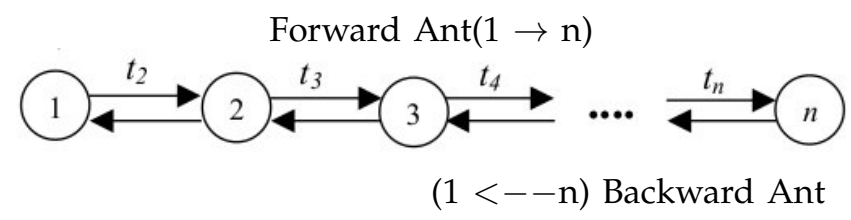

Figure 3. Forward and backward ant's path

path of the forward ant by popping the stack, making modifications in the routing tables at each intermediate node according to the following learning rules:

$$
\begin{aligned}
& \text { IF (node was in the path of the ant) } \\
& \text { THEN } \quad p^{\text {new }}(i)=p^{\text {old }}(i)+r\left[1-p^{\text {old }}(i)\right] \\
& \text { ELSE } \quad p^{\text {new }}(i)=p^{\text {old }}(i)-r p^{\text {old }}(i)
\end{aligned}
$$

where $r \in(0,1]$ is the reinforcement factor which is central to express path quality. The reinforcement factor should be a factor of trip time of the node neighborhood. This factor is given by the relationship $r=t_{1} / t_{2}$, where $t_{1}$ is the minimum trip time of all the forward ants, and $t_{2}$ is the trip time of the current forward ant from a node to the destination node.

$p^{\text {new }}(i)$ is the new probability and $p^{\text {old }}(i)$ is the old probability for a node $i$ in the routing table. Equation 2 always increases the probability $p^{\text {new }}(i)$, what means that every update is a positive feedback. This resembles the pheromone of the ants where every dropped 
pheromone increases the strength of a pheromone trail but not weakens it.

The reinforcement factor ensures that the probability increase is inversely proportional to the travel time of the forward ant. The higher the time $t_{2}$, the lower the probability and the lower the time $t_{2}$, the higher the probability. By that, good paths receive a strong update while bad paths receive a low update only.

For a given value of $r$, the absolute and relative increase of $p^{\text {new }}(i)$ is much larger for small values of $p^{\text {old }}(i)$ than for large values of $p^{\text {old }}(i)$. By that weighted change, low probabilities go up very fast to adapt to the new traffic situation, whereas probabilities which are already high are increased only a little bit.

Whenever a probability in the routing table is increased by equation 2 , it is necessary to ensure that the sum of the probabilities in each row remains 1. Equation 3 is used to normalize the values. This means that a probability can only decrease if another probability in the same row increases. A probability can approach zero if other probabilities in the same row of the routing table increase much more often or much stronger. This would mean that a forward ant hardly takes the route associated with this low probability, what means that this probability receives nearly no further updates. But indeed, this route can become the fastest route in time under changed traffic conditions. After that, the probabilities per destination are normalized again for all the nodes, so that the sum of the probabilities is 1 , i.e. $\sum_{i} P_{i}=1$ which ensures that every link is used by forward agents from time to time and so new routes are explored regularly.

The value of a probability in a routing table finally depends on two facts: the trip times experienced by the forward ants that use the route associated with the probability and the frequency of the updates (the number of ants that use the route). For example, if the trip times are very low, the reinforcement factor $r$ is very high and so the probability is strongly increased with every update. So the probability will be very high even if the frequency of the update is low. But on the other hand, also high trip times, which mean a low reinforcement factor $r$, can lead to a high probability if the frequency of the updates is high. In that way, high frequent updates with low travel times lead to the highest probabilities.

\subsection{Proposed Algorithm - AntNet-LA}

We propose to use a passive localization algorithm to calculate the location of a node based on square of difference between the node co-ordinates.

Let $n_{i}=\left[x_{i}, y_{i}, z_{i}\right]$ denote the $(x, y, z)$ coordinate of the $\mathrm{i}^{\text {th }}$ node, $n_{s}=\left[x_{s}, y_{s}, z_{s}\right]$ denote the co-ordinates of source node, and $n_{d}=\left[x_{d}, y_{d}, z_{d}\right]$ denote co-ordinates of destination nodes. Let $R_{i s}$ and $R_{d s}$ be the measured distances between a node with unknown location, $n_{i}$, and the nodes $n_{s}$ and $n_{d}$ respectively. The range difference in the distance of nodes $n_{s}$ and $n_{d}, r_{s d}$, is equal to:

$$
r_{s d}=R_{i s}-R_{d s}=\left\|n_{i}-n_{s}\right\|^{2}-\left\|n_{d}-n_{s}\right\|^{2}
$$

There are

$$
\left(\begin{array}{l}
n \\
r
\end{array}\right)=\frac{N !}{(N-2) ! 2 !}
$$

distinct range differences. However, all of these range measurements can be completely determined from (1) range measurements. The square of the distance between the source and the $\mathrm{i}^{\text {th }}$ node can be written as:

$$
R_{i s}^{2}=\left(r_{i d}+R_{d s}\right)^{2}=r_{i d}^{2}+2 R_{d s} r_{i d}+R_{d s}^{2}
$$

We also have:

$$
R_{i s}^{2}=\left\|n_{i}-n_{s}\right\|^{2}=n_{i}^{2}-2 n_{i} n_{s}^{T}+n_{s}^{2}
$$

If we set the left terms of equations 5 and 6 to be equal to each other, we have:

$$
2 n_{i} n_{s}^{T}=n_{i}^{2}+n_{s}^{2}-r_{i d}^{2}-2 R_{d s} r_{i d}-R_{d s}^{2}
$$

For $i=d$, we can rewrite equation 7 as:

$$
2 n_{i} n_{s}^{T}=n_{d}^{2}-n_{s}^{2}-R_{d s}^{2}
$$

Subtracting equation 8 from equation 6 we have:

$$
2\left(n_{i}-n_{d}\right) n_{s}^{T}=n_{i}^{2}-n_{d}^{2}-r i d^{2}-2 R_{d s} r_{i d}
$$

Equation 9 can also be written in matrix form as:

$$
A_{d} n_{s}^{T}=B_{d}-R_{d s} C_{d}
$$

Where

$$
\begin{gathered}
A_{d}=\left(\begin{array}{ccc}
\left(x_{i}-x_{d}\right) & \left(y_{i}-y_{d}\right) & \left(z_{i}-z_{d}\right) \\
\vdots & \vdots & \vdots \\
\left(x_{d-1}-x_{d}\right) & \left(y_{d-1}-y_{d}\right) & \left(z_{d-1}-z_{d}\right) \\
\left(x_{d+1}-x_{d}\right) & \left(y_{d+1}-y_{d}\right) & \left(z_{d+1}-z_{d}\right) \\
\vdots & \vdots & \vdots \\
\left(x_{N}-x_{d}\right) & \left(y_{N}-y_{d}\right) & \left(z_{N}-z_{d}\right)
\end{array}\right) \\
B_{d}=\frac{1}{2}\left(\begin{array}{c}
\left(x_{i}^{2}-x_{d}^{2}-r_{1 d}^{2}\right) \\
\vdots \\
\left(n_{d-1}^{2}-n_{d}^{2}-r_{d-1 j}^{2}\right) \\
\left(n_{d+1}^{2}-n_{d}^{2}-r_{d+1 j}^{2}\right) \\
\vdots \\
\left(n_{N}^{2}-n_{d}^{2}-r_{N d}^{2}\right)
\end{array}\right) 1(N-1) \\
\left(\begin{array}{c}
\left(r_{i d}\right) \\
\vdots \\
\left(r_{d-1 j}\right) \\
\left(r_{d+1 j}\right) \\
\vdots \\
\left(r_{N d}\right)
\end{array}\right) \\
C_{d}=(10 \mathrm{c}) \\
1(N-1)
\end{gathered}
$$

A closed-form solution that is useful for some subsequent derivations is given by:

$$
n_{s}^{T}=\left(A_{d}^{T} A_{d}\right)^{-1} A_{d}^{T}\left(B_{d}-R_{d s} C_{d}\right)
$$

For the given measurements $\left\{r_{\mathrm{i} d}, i=1, \ldots, N\right\}$, the vectors $B_{d}$ and $C_{d}$ are known. So matrix $A_{d}$ which depends only on the node locations. (assumed to be known. We use a custom function to store node coordinates in our algorithm). The (10a), (10b), (10c), (10d) are the location vectors $n_{s}$ and the distance $R_{d s}$ between 
a source and destination node. It is the presence of $R_{d s}$ which complicates the solution of (10).

In order to eliminate the "nuisance parameter" $R_{d s}$ by pre-multiplying equation 10 a by a matrix $M$ which has $C_{d}$ in its null shape. The matrix

$$
M_{d}^{k}=\left(I-Z^{k}\right) D_{d}
$$

where

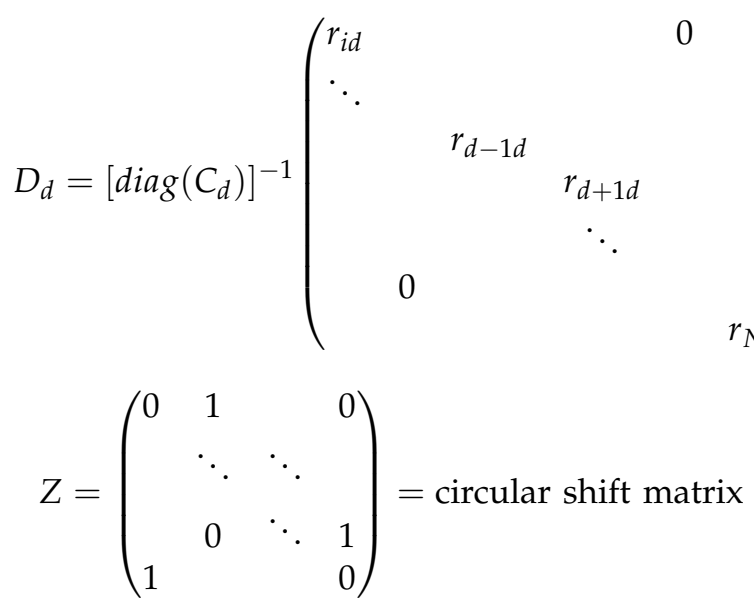

$$
\begin{aligned}
& D_{d C d}=1 \triangleq[1,1 \ldots, 1]^{T}
\end{aligned}
$$

and

$$
\left(I-Z^{k}\right) 1=1-Z^{k} 1=1-1=0
$$

To simplify subsequent equation we assume that $K=1$ and suppress the subscript in $M_{d}^{k}$. It shows that

$$
M_{d} S_{d} n_{s}=M_{d} B_{d}
$$

The location vector $n_{s}$ can now be computed by using good least squares equation solver on the linear set of equation 15. A closed form of solution is given by

$$
n_{s}=\left(S_{d}^{T} M_{d}^{T} M_{d} S_{d}\right)^{-1} S_{d}^{T} M_{d}^{T} M_{d} B_{d}
$$

For matrix $A_{j}$ to be non-singular it is necessary that the number of rows of $A_{j}$ be larger than number of columns i.e. $(N-1)>n$, where $n$ is the dimension of $n_{s}$ (2dimension, 3-dimension etc.). Thus for unique solution of equation 16 to exist we must have $N>(n+1)$. Thus by solving equation 16 , we can find the coordinates of the source node. Hence from this source node packet is transmitted to $\mathrm{i}^{\text {th }}$ and $\mathrm{d}^{\text {th }}$ node. Using these $\mathrm{i}^{\text {th }}$ and $\mathrm{d}^{\text {th }}$ nodes as source nodes next neighboring node coordinates are found and packets are sent to these neighboring nodes. In order to minimize computation and memory resources we have considered $z$ coordinates of all nodes equal to zero. Hence in our case $n=2$ and number of nodes $(N)>3$.

Algorithm 1 shows our proposed algorithm.

\section{Working of AntNet-LA}

In order to explain the working of AntNet-LA, we consider an example of a network which consists of 8 nodes. This network is shown in figure 4 . We make following assumptions in relation with the example:

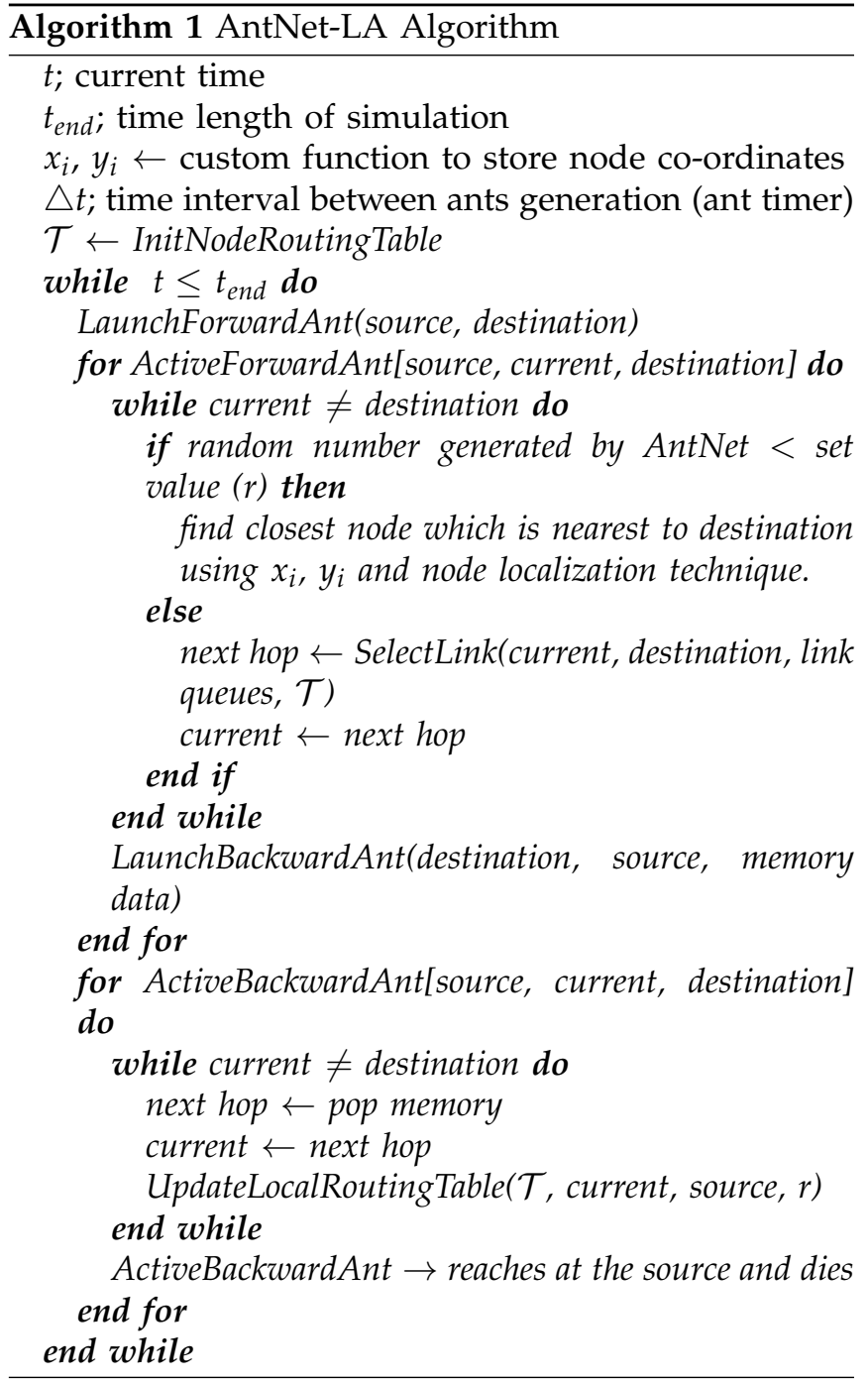

1) Each node is having information about the coordinates of every other node in the network. We have used a custom defined function in our algorithm to store the co-ordinate values.

2) The datagram packets are required to be transmitted from the source node, denoted by 1 to the destination node, denoted by 6 .

3) Initially all nodes in the network have equal probability of routing.

4) Initially all nodes are stationary.

5) Later all nodes moves at an average velocity of $4 \mathrm{~m} / \mathrm{s}$ to $10 \mathrm{~m} / \mathrm{s}$.

The packet transmission takes place in the following way:

1) As shown in Figure 4, the data packet from node 1 is forwarded randomly if the original AntNet algorithm would have followed. But AntNet-LA calculates the co-ordinates $n_{i}$ using equation 11 . If these calculated co-ordinates are closer to the that of node 2 , and if the probability ( $r$ factor) is less than the pre-setted value then the node 1 forwards the packet to the node 2 as shown in Figure 5(a). Hence this path is said to be optimum.

2) Now co-ordinates of the node 2 becomes $n_{s}$. The algorithm now calculates new values of $n_{i}$ using 


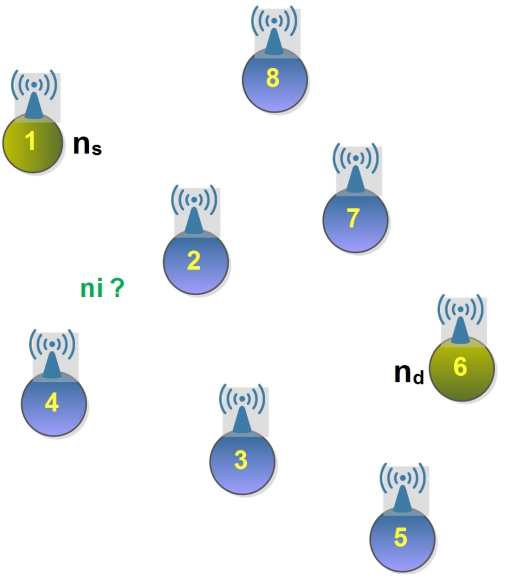

Figure 4. A network with 8 nodes

equation 11 and if these calculated co-ordinates are closer to the that of node 7 and if the probability ( $r$ factor) is less than the pre-setted value then node 2 forwards the packet to the node 7 as shown in Figure 5(b).

3) By following similar procedure above the packet is finally delivered to the node 6 as shown in figure 5(c).

4) Once the packet is delivered to the destination the forward ants gets converted in backward ants. These backward ants follows the same path as forward ants. These backward ants updates the routing table at every node. Hence the $r$ factor is re-calculated during routing table updates. The backward ant vanishes when it reaches the source node.

5) For the transmission of next packets the new values of probabilities ( $r$ factors) are considered. Hence the cycle repeats with these new probability values.

Therefore the AntNet-LA algorithm helps ants to find the optimum path for packet transmission. This algorithm avoids the randomness of AntNet algorithm, where the next hop for packet transmission is entirely depend upon the probability value.

\section{Deployment of AntNet-LA Algorithm}

Mobile nodes (which are both hosts and routers) use an ad hoc routing protocol to route packets. AntNetLA protocol along with Internet Protocol (IP) can be deployed at network layer as shown in figure 6 . The modulation techniques like frequency hopping spread spectrum (FHSS) can be used in IEEE 802.11 physical layer.

The Logical Link Control (LLC) sub-layer must perform following functions:

1) Manage the communication on the data link.

2) Link addressing with AntNet-LA and IP.

3) Defining Service Access Points (SAPs)

A SAP must be a kind of port used by the network layer protocol to access services provided by the data

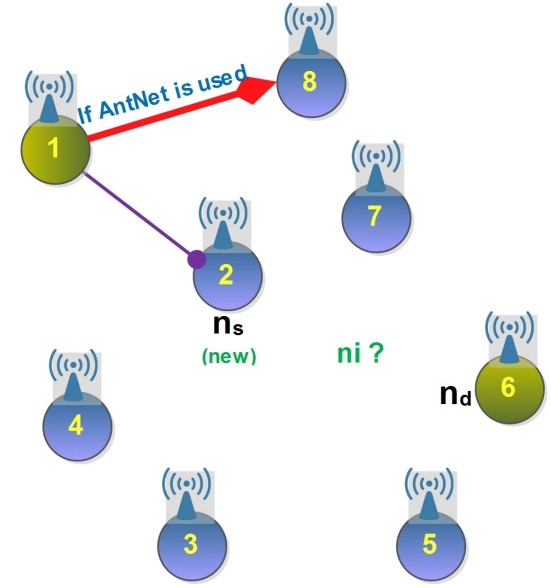

(a) Packet transmission from node 1 to node 2

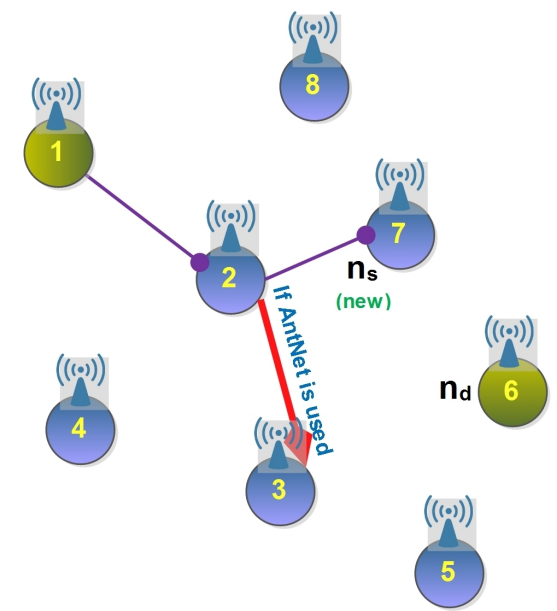

(b) Packet transmission from node 2 to node 7

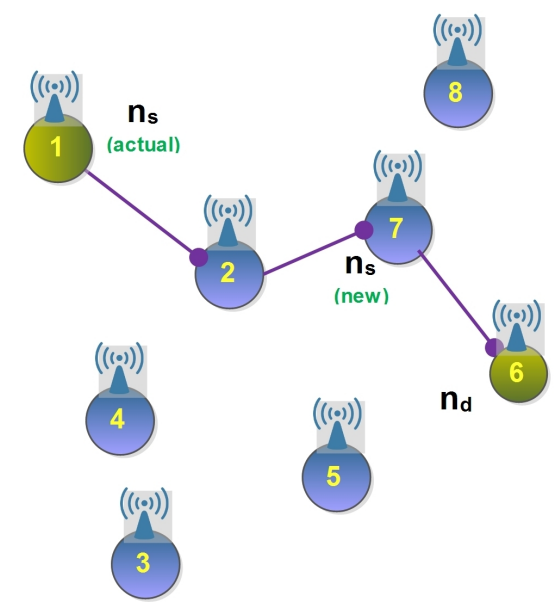

(c) Packet transmission from node 7 to node 6

Figure 5. Example of 8 nodes

link layer. The SAP must act as an identifier for the user of data link layer.

The main function of Media Access Control (MAC) sub-layer is to co-ordinate and regulate the data transmission of active nodes. This co-ordination of data transmission between multiple active nodes is the responsibility of MAC, which must be implemented as part of MAC sub-layer in each transmitting node. 
Table IV

Simulation Parameters for Comparison between AODV, AOMDV, DSR AND DSDV PRotocols

\begin{tabular}{|l|l|}
\hline Parameters & Value \\
\hline Routing Protocols & $\begin{array}{l}\text { AntNet-LA, AntNet, AODV, } \\
\text { AOMDV, DSR, DSDV }\end{array}$ \\
\hline Packet Size & 64 bytes \\
\hline Area & $800 \mathrm{~m} \times$ 800m \\
\hline No. of nodes & 50 \\
\hline Mobility Model & Disaster Area (4 groups) \\
\hline Data Traffic & CBR \\
\hline$r$ - factor & $0.1-0.9$ \\
\hline $\begin{array}{l}\text { For Comparison between } \\
\text { LAR, GeoAODV, PBANT and } \\
\text { AntNet-LA } r \text { - factor }\end{array}$ & 0.9 \\
\hline Ant Timer & $0.1-100$ seconds \\
\hline Avg. Transmission Range & 400 meters. \\
\hline Simulation Time & 180 seconds. \\
\hline
\end{tabular}

Table $\mathrm{V}$

Simulation Parameters for Comparison between AntNet-LA, LAR, GEOAODV aNd PBANT Protocols

\begin{tabular}{|l|l|}
\hline Parameters & Value \\
\hline Routing Protocols & $\begin{array}{l}\text { AntNet-LA, LAR, GeoAODV } \\
\text { and PBANT }\end{array}$ \\
\hline Packet Size & 64 bytes \\
\hline Area & $800 \mathrm{~m}$ x $800 \mathrm{~m}$ \\
\hline No. of nodes & 50 \\
\hline Mobility Model & Disaster Area (4 groups) \\
\hline Data Traffic & CBR \\
\hline $\begin{array}{l}r-\text { factor (for AntNet-LA and } \\
\text { PBANT) }\end{array}$ & 0.9 \\
\hline $\begin{array}{l}\Omega_{1}, \Omega_{2}, \Omega_{3} \text { (wait parameter for } \\
\text { PBANT) [7] }\end{array}$ & $1.25,1$ and 0.75 respectively [7] \\
\hline $\begin{array}{l}v_{1} \quad v_{2}, \quad \text { (amount of } \\
\text { pheromone to be deposited } \\
\text { based on zone formation for } \\
\text { PBANT) [7] }\end{array}$ & 20,1 and 1 respectively [7] \\
\hline Avg. Transmission Range & 400 meters. \\
\hline Simulation Time & 180 seconds. \\
\hline
\end{tabular}

\section{Performance Evaluation}

\subsection{Simulation Setup}

The performance of AntNet-LA algorithm was evaluated through extensive simulations using Network Simulator (NS) version 2.34 on Ubuntu ${ }^{\circledR}$ operating system. We have used Tracegraph [14], awk, c-programming languages and shell scripts to obtain the results. Table IV and V shows simulation parameters for our experimentations. Extensive simulations were carried out by varying $r$ factor and by varying ant timer. We also

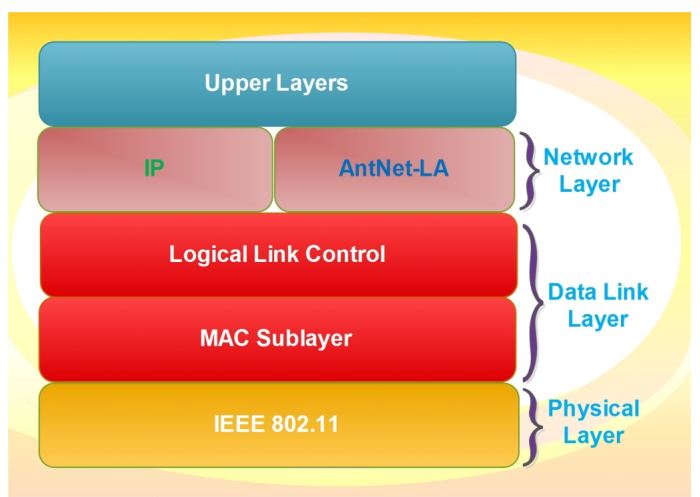

Figure 6. Deployment of AntNet-LA in the OSI protocol stack

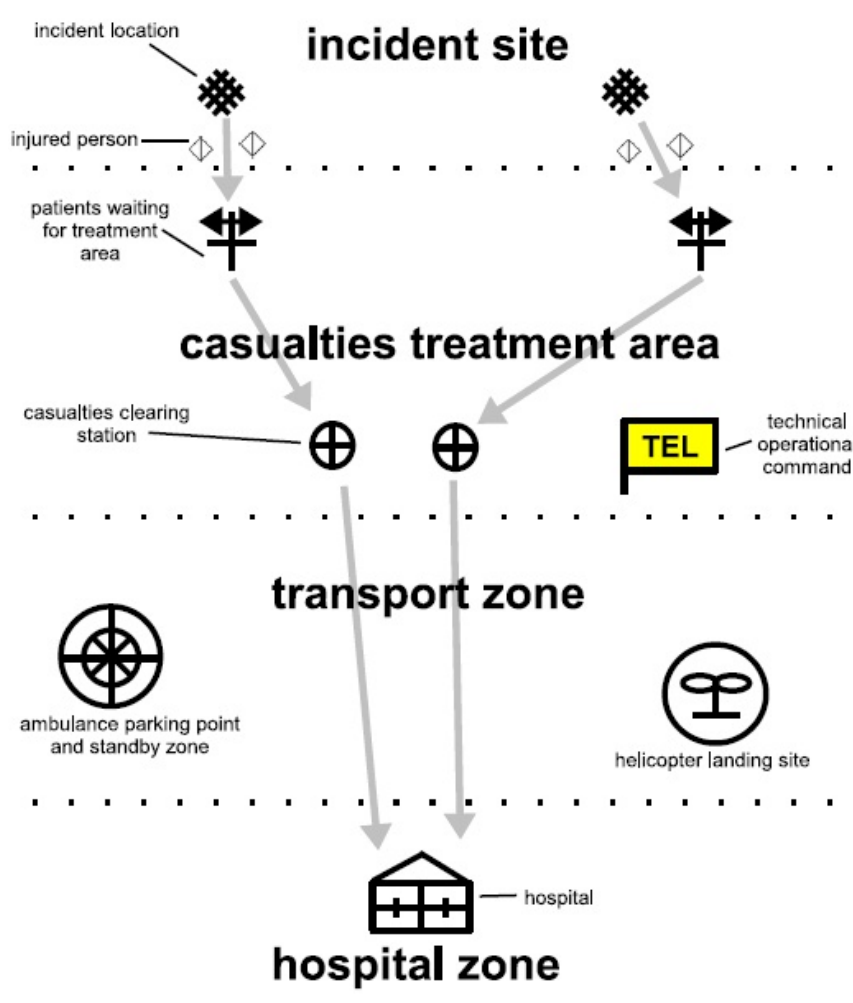

Figure 7. A typical disaster area mobility model [16]

compare performance of AntNet-LA with Antnet. We compare our results with AntNet [13], Ad-hoc On Demand Distance Vector (AODV) [5], Ad-hoc On Demand Multipath Distance Vector (AOMDV), Dynamic Source Routing (DSR), Destination-Sequenced Distance Vector Routing (DSDV) [15] protocols with our proposed one.

\subsection{Disaster Area Mobility model}

In Disaster Area mobility model, the disaster area and its surrounding is divided into different areas: incident site, casualties treatment area, transport zone, and hospital zone [16] as shown in Figure 7. The incident site is the place where the disaster actually happened. In this area affected and injured people as well as fatalities are found and the disaster (e.g. fire) has to be minimized. The affected and injured people are brought to the casualties treatment area. The casualties treatment area consists of two places: the patients waiting for treatment area and the casualties clearing station. The people are rescued from danger and wait there for their treatment. Then they are transported to the casualties clearing station which is still within the disaster area. After an extended first aid they are transported to hospital. The transport zone is an area where transport units (ambulance coaches and rescue helicopters) wait in stand-by areas to take these people to hospitals. The technical operational command is usually located in the casualties treatment area as well.

The areas beside the incident location (e.g. places where tents are set up) are chosen by humans such that there are no obstacles inside these areas. At the incident location the units (e.g. firefighters) will destroy larger hindering obstacles. Smaller ones can be ignored, 
because they only have little impact on the movement. So there are only obstacles between different areas. Thus, they only affect transport units. For the transport between the patients waiting for treatment area and the casualties clearing station, there are typically transport troops. These troops are pedestrians e.g. four that carry a patient on a barrow. These troops pick up a patient and transport him on the direct way to his destination. Thus, they choose the optimal (shortest) paths avoiding obstacles. The hospitals are typically far away and not part of the disaster area communication network. The vehicles of the transport zone (e.g. ambulance coaches) transport the patients to the hospital. Thus, these transport units typically arrive and leave the network perpetually.

\subsection{Parameters for Performance Analysis}

The performance was evaluated by comparing the results of the AntNet-LA algorithm with Antnet [17]. The considered performance indices were as follows [8, 18]:

1) Average End-to-End Delay (seconds):

This includes all possible delay caused by buffering during route discovery latency, queuing at the interface queue, retransmission delay at the MAC, propagation and transfer time. It is defined as the time taken for a data packet to be transmitted across an ad-hoc network from source to destination.

$D=\left(T_{r}-T_{s}\right)$ Where $T_{r}$ is receive Time and $T_{S}$ is sent Time.

2) The number of lost packets:

These packets are lost either at source node or at destination node or at intermediate node. More the loss packets lesser the packet delivery ratio.

3) Jitter:

The term jitter is often used as a measure of the variability over time of the packet latency across a network. A network with constant latency has no variation (or jitter).

4) Route Cost:

It is the ratio of routing bytes to CBR bytes. Routing Cost $=N_{\text {route }} / N_{c b r}$.

5) Throughput (kb/second):

It is the rate at which network send or receive data. It is rated in term of number of bits per seconds. It is the sum of data rates that are delivered to all nodes in a network.

\section{Results AND Discussion}

\subsection{Results}

8.1.1 Comparison between AntNet, AntNet-LA, AODV, $A O M D V, D S R$ and DSDV protocols: Whenever any disaster occurs, it is very important that injured people must get proper treatment in time. For that injured people must reach the hospital in minimum time. It is also important to control the traffic on road, so that patients can reach the hospital in time.

- From Figure 8(a) we can see that avg. end to end delay is high at the beginning of the simulation

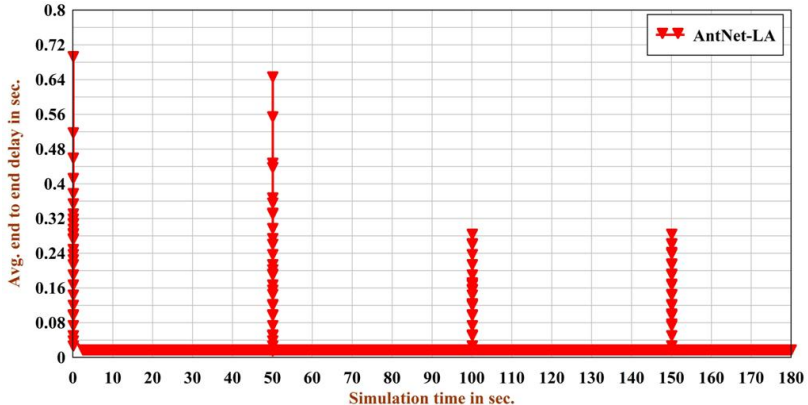

(a) Avg. end to end delay Vs. Simulation time for ant timer $=50$ sceconds and $r$ factor $=0.9$. for AntNet-LA

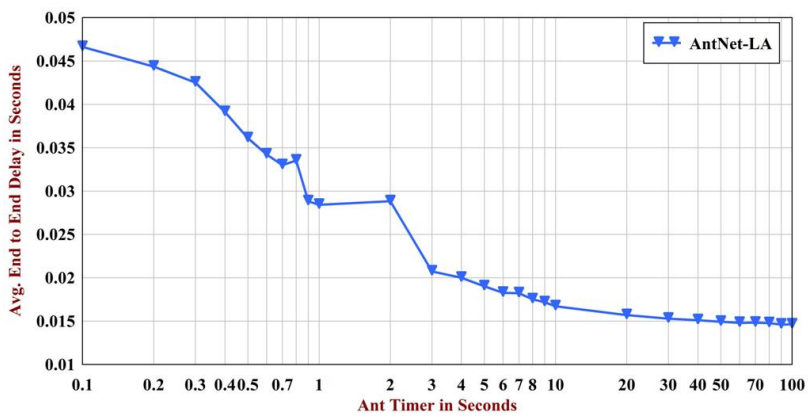

(b) Avg. end to end delay Vs. ant timer for AntNet-LA

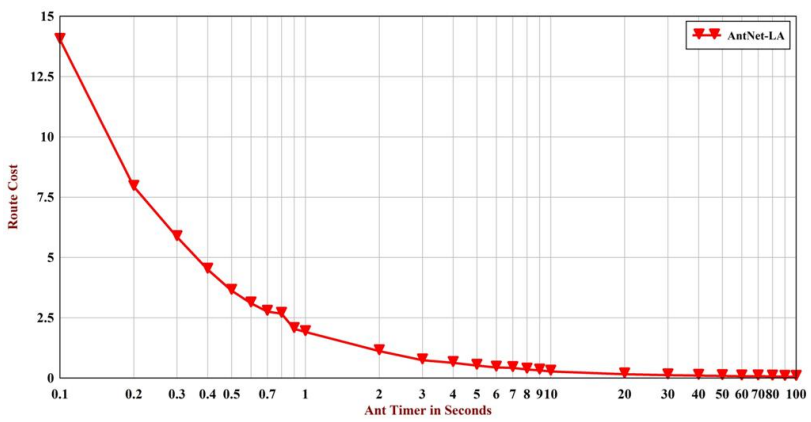

(c) Route cost Vs. ant timer for AntNet-LA

Figure 8. Different QoS parameters for AntNet-LA

and it reduces as simulation period progresses. It is because at the beginning, the patients were transferred to "casualties treatment area" where first aid treatment was given to patients. Later on the patients were moved quickly to the hospital through "transport zone".

- Ant timer specifies the time required to complete the algorithm. From Figure 8(b) we can see exponential curve of avg. end to end delay. Hence in order to minimize end to end delay, we must allow more time to complete the algorithm.

- From Figure 8(c) we can see that as period of ant timer increases the route cost decreases. It is because by allowing more time to complete the algorithm, the number of rescue workers (ant packets) required to help affected people decreases.

- From Figure 9(a) we can see that two packets were lost at the receive node and five packets were lost at the send node. This means that two injured persons died after reaching the hospital. Five injured persons died before reaching hospital due to delay in the treatment.

- Figure 9(b) shows jitter of received packets. The 


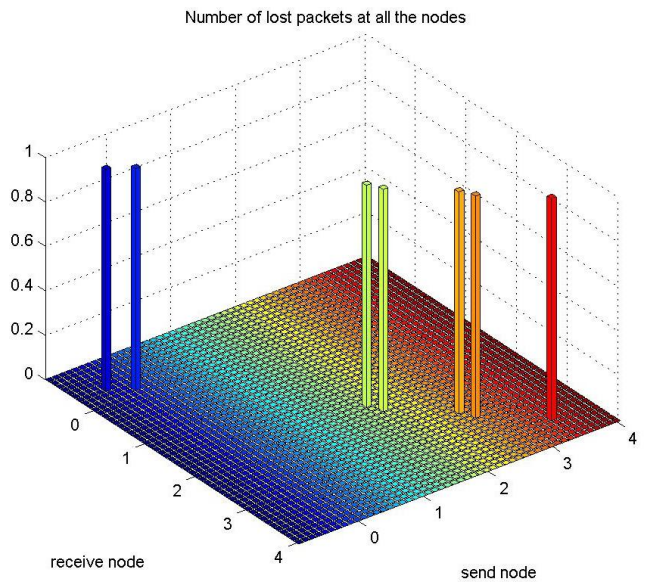

(a) Number of lost packets for AntNet-LA

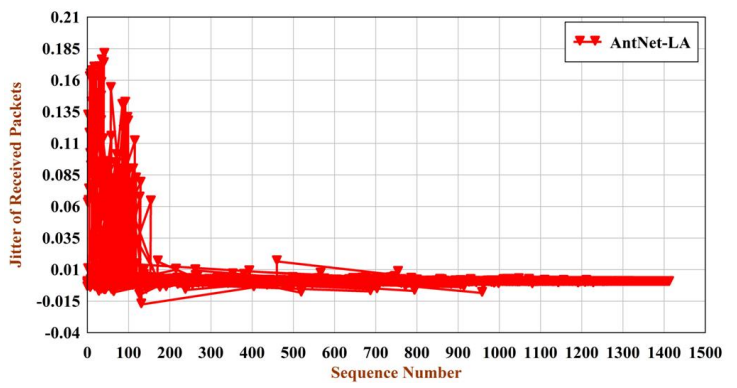

(b) Jitter of received packets Vs. Sequence number of packets

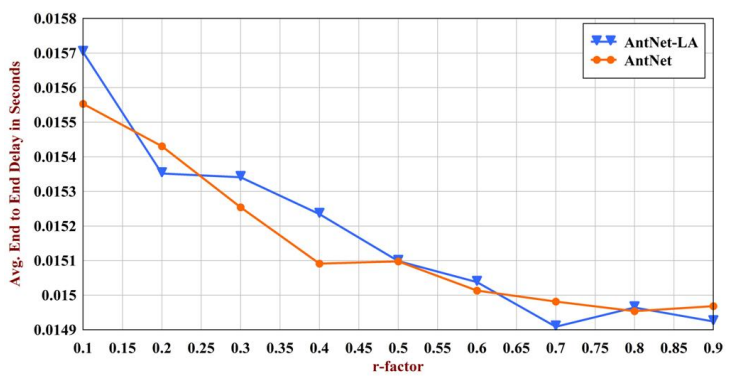

(c) Average end to end delay for AntNet-LA and AntNet Vs. different $r$ factors for ant timer $=50$ seconds.

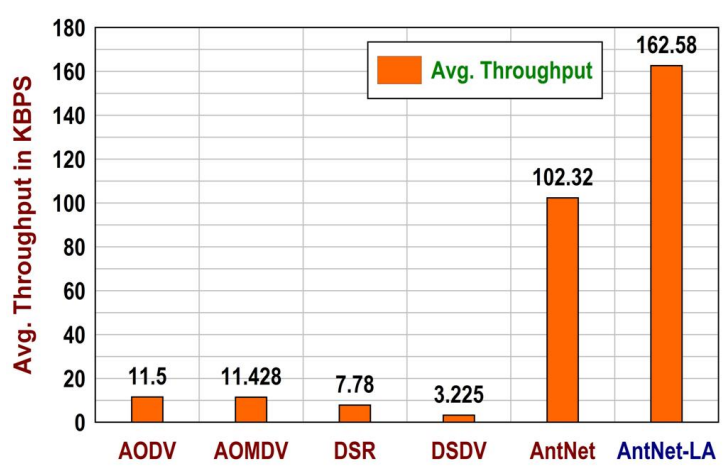

(d) Avg. throughput for AntNet-LA and AODV, AOMDV, DSR and DSDV protocols

Figure 9. Different QoS parameters for AntNet-LA, AntNet, AODV, AOMDV, DSR and DSDV protocols

jitter graph is mainly on the positive side of $y$ axis. This indicates that the variation in end to end delay is less which means that all injured persons reached the hospital in minimum time.

- From Figure 9(c) we can see that the end to end

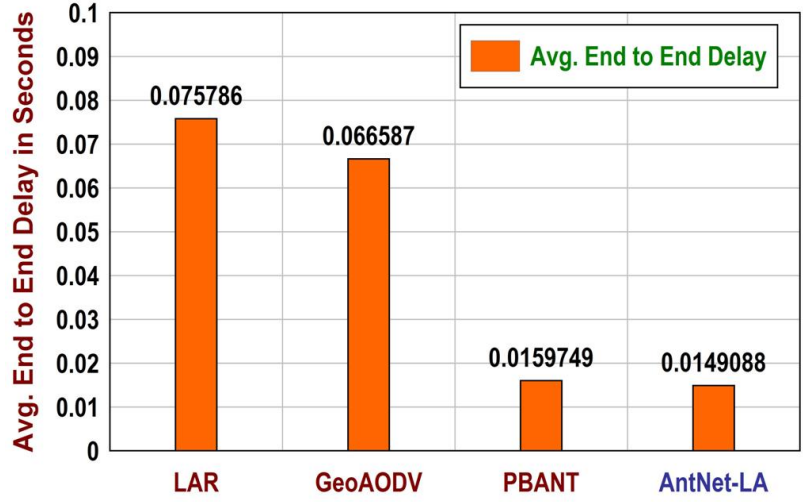

(a) Avg. end to end delay

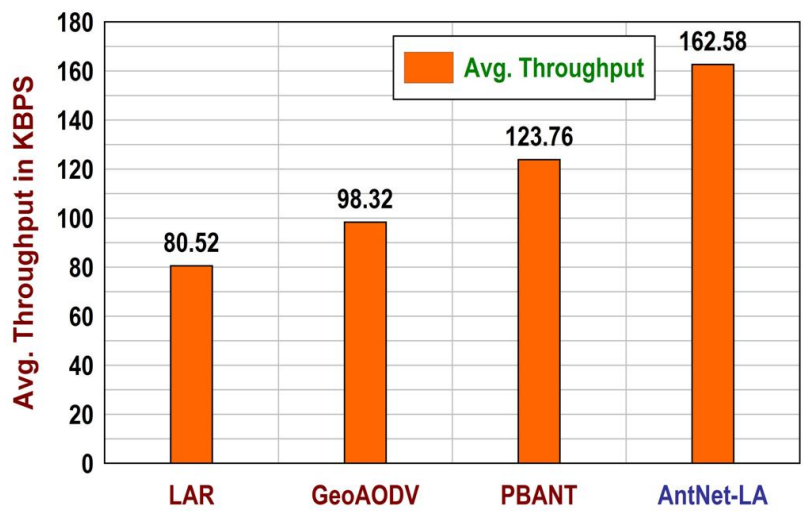

(b) Avg. throughput

Figure 10. Different QoS parameters for AntNet-LA, LAR, GeoAODV and PBANT protocols

delay with AntNet-LA and $r$ factor $=0.25$ to 0.6 is higher as compared with AntNet for the same $r$ factors. The end to end delay for AntNet-LA with $r$ factors $=0.63$ to 0.8 and 0.81 to 0.9 is lower as compared with AntNet for the same $r$ factors.

- Whenever any disaster occurs it is important that every injured person must reach to the hospital in time. Avg. throughput can be mapped as number of patients who reaches the hospital per unit time. From Figure 9(d) we can see that avg. throughput for AntNet-LA is higher than other protocols. This means that the number of injured persons per unit time reaching the hospital using AntNet-LA protocol are higher than the number of persons per unit time reaching to the hospital with other protocols.

8.1.2 Comparison with other Location Aware Protocols: The proposed protocol is compared with other location aware protocols such as LAR [4], GeoAODV [6] and PBANT [7]. LAR and GeoAODV protocols are implemented using OPNET Modeller [19, 20]. PBANT is implemented using Network Simulator - 2.34 [21]. Simulation results are presented in figures 10 (a) and 10(b). Simulation results shows that AntNet-LA performs better than LAR, GeoAODV and PBANT protocols in terms of avg. throughput. The avg. end to end delay for AntNet-LA is marginally better than PBANT. 


\subsection{Discussion}

In AntNet-LA, the continual on-line construction of the routing tables is the emergent result of a collective learning process. Each forward-backward agent pair is complex enough to find good route and to adapt the routing tables for a single source-destination path. It is the interaction between the agents that determines the emergence of a global effective behavior from the network performance point of view. Ants co-operate in their problem solving activity by communicating in an indirect way. Good routes are discovered by forwarding the ants to the closest next hop node using location awareness algorithm. This communication paradigm, called stigmergy matches well the intrinsically distributed nature of the routing problem. Cooperation among agents goes on at two levels: (a) by modifications of the routing tables, and (b) by modifications of local models that determine the way the ants' performance is evaluated. Modifications of the routing tables directly affect the routing decisions of following ants towards the same destination, as well as the routing of data, which, in turn, influences the rate of arrival of other ants towards any destination. In this perspective, once the interface with the node's data structure is defined, the internal policy of the agents can be transparently updated. In the following we try to justify AntNet-LA performance by highlighting some of its characteristics and by comparing them with those of the competing algorithms. We focus on the following main aspects:

- The information AntNet-LA maintains at each node is more complete and organized in a less critical way than that managed by the other algorithms.

- AntNet-LA uses probabilistic routing table which depends on the distance between nodes which is unlike to the Antnet algorithm which uses simple probabilistic routing table. This technique is having double positive effects of better redistributing data traffic on alternative routes and of providing ants with a built-in exploration mechanism.

- In AntNet-LA, we experimentally observed the robustness to changes in the ants' generation rate: for a wide range of generation rates, the algorithm performance is very good and the routing cost is almost negligible.

- Even though the computing complexity of AntNetLA is high which is because of the complexity in solving the matrix of equation 16, this algorithm is less dependent on external parameters as compared with other location aware protocols.

\subsection{Advantages of AntNet-LA over other Location Aware Protocols}

- Even though computing complexity of AntNet-LA is high, it requires only one parameter to be set i.e $r$ factor. Whereas

- PBANT requires $\Omega_{1}, \Omega_{2}$ and $\Omega_{3}$ to be set. PBANT also requires $v_{1}, v_{2}$ and $v_{3}$ to be set. These parameters have significant impact on performance of PBANT [7]. It is very difficult to obtain exact parameters in order to satisfy required QoS.

- LAR requires formation of "expected zone" and "request zone". The formation of these zones may vary widely if nodes in the network moves at a higher speed.

- AntNet-LA requires only "Ant" packets to establish the route. Whereas GeoAodv requires route request (RREQ) and route reply (RREP) packets to establish and maintain the routes [19]. Hence control overheads of GeoAODV are more than that of AntNet-LA.

\section{Possible Applications of AntNet-LA Algorithm}

1) AntNet-LA can be applied for packet transmission in the datagram networks. In high-speed datagram networks QoS is very important. This means that a user session can specify the QoS it needs to receive from the network. For example it should specify the maximum and minimum levels of throughput, or end to end delay or the minimum packet delivery ratio.

2) AntNet-LA can be applied to the network whose arrival rate is dictated by the probabilistic model of the algorithm.

3) AntNet-LA can be applied to the static model where all the sessions start at the beginning of the transmission stage and they last until the end.

\section{Conclusions and Future Work}

In this paper we have obtained the performance of AntNet-LA protocol for ad hoc network that is based on mobile software agents modelled on ants. Ants are used to collect and disseminate information about location of nodes using range difference method. This is a key aspect of AntNet-LA that helps to accelerate route discovery. The combination of these principles provide a better routing algorithm. Simulation results shows that by selecting proper value of reinforcement factor $r$ lower end to end delay as compared with AntNet protocol can be obtained. Our simulation results also shows that AntNet-LA performs better than other conventional and distance aware protocols

In future AntNet-LA can be modified by applying clustering concept. This concept may help in minimizing energy consumption. Aslo in future performance of AntNet-LA can be analyzed using other mobility models like Group and Random Direction. Our approach can be utilized for applications based on peer-to-peer communications [22].

\section{ACKNOWLEDGMENT}

The author would like to thank the reviewers for their detailed comments on earlier versions of this paper. 


\section{REFERENCES}

[1] G. Caro and M. Dorigo, "AntNet - distributed stigmergetic control for communications networks," Journal of Artificial Intelligence Research, vol. 9, pp. 317-356, 1998.

[2] P. Lalbakhsh, B. Zaeri, and M. N. Fesharaki, "Applying nonlinear learning scheme on antnet routing algorithm," in Proc. Annual Meeting of the North American Fuzzy Information Processing Society (NAFIPS), 2010, pp. 1-6.

[3] S. Kamali and J. Opatrny, "POSANT: a position based ant colony routing algorithm for mobile ad-hoc networks," in Third International Conference on Wireless and Mobile Communications. ICWMC '07., 2007, pp. 21-21.

[4] Y. Ko and N. Vaidya, "Location-aided routing (LAR) in mobile ad hoc networks," Wireless Networks, vol. 6, no. 4, pp. 307-321, November 2000.

[5] C. Perkins, E. B. Royer, and S. Das, "Ad hoc on-demand distance vector (AODV) routing," RFC 3561, july 2003.

[6] H. Asenov and V. Hnatyshin, "GPS-enhanced AODV routing," in Proc. of the 2009 International Conference on Wireless Networks (ICWN'09), 2009.

[7] B. R. Sujatha and V. P. Harigovindan, "Performance analysis of PBANT (PBANT: Position based ant colony routing algorithm) for manets," in Proc. 6th IEEE International Conference on Networks (ICON), 2008, pp. 1-6.

[8] E. Heidari, "Intelligent clustering in wireless sensor networks," in First International Conference on Networks and Communications, 2009. NETCOM '09, 2009, pp. 12-17.

[9] S. N. Sivanandam and S. N. Deepa, Introduction to Genetic Algorithms. Heidelberg: Springer-Verlag, 2008, pp. 410422.

[10] V. Jha, K. Khetarpal, and M. Sharma, "A survey of nature inspired routing algorithms for manets," in Proc. 3rd IEEE International conference on Electronics Computer Technology (ICECT), april 2011, pp. 16-24.

[11] G. Di Caro, F. Ducatelle et al., "Anthocnet: an adaptive nature-inspired algorithm for routing in mobile ad hoc networks," Special Issue on Self-organisation in Mobile Networking, vol. 16, pp. 443-465, 2004.

[12] A. Mora, J. Merelo, C. Millan, J. Torrecillas, J. Laredo, and P. Castillo, "Enhancing a MOACO for solving the bi-criteria pathfinding problem for a military unit in a realistic battlefield," Applications of Evolutionary Computing, pp. 712-721, 2007.

[13] G. Di Caro and M. Dorigo, "Antnet: Distributed stigmergetic control for communications networks," Journal of Artificial Intelligence Research (JAIR), vol. 9, pp. 317-365, 1998.

[14] K. Lukoit, N. Wilde, S. Stowell, and T. Hennessey, "Tracegraph: immediate visual location of software features," in Proc. International Conference on Software Maintenance, august 2002, pp. 33-39.

[15] G. R. Vijayavani and G. Prema, "Performance comparison of MANET routing protocols with mobility model derived based on realistic mobility pattern of mobile nodes," in IEEE International Conference on Advanced Communication Control and Computing Technologies (ICACCCT), aug. 2012, pp. $32-36$.

[16] N. Aschenbruck, E. Gerhards-Padilla, M. Gerharz, M. Frank, and P. Martini, "Modelling mobility in disaster area scenarios," in Proceedings of the 10th ACM Symposium on Modeling, analysis, and simulation of wireless and mobile systems, ser. MSWiM '07. New York, NY, USA: ACM, 2007, pp. 4-12.

[17] Z. Lin and L. Xiaoping, "The research and improvement of AntNet algorithm," in Proc. 2nd Int Informatics in Control, Automation and Robotics (CAR) Asia Conf, vol. 2, 2010, pp. 505-508.

[18] V. Godbole, "Performance analysis of bio-inspired routing protocols based on random waypoint mobility model," Defence S E T Technical Bulletin, Research $\mathcal{E}$ Technical Institute of Defence, Govt. of Malesia, vol. 5, no. 2, pp. 114-134, November 2012.
[19] H. Asenov and V. Hnatyshin, "Design and implementation of an opnet model for simulating geoaodv manet routing protocol," in Proc. of OPNETWORK Wireless Ad Hoc and Wireless Personal Area Networks, 2010.

[20] H. Asenov, V. Hnatyshin, and J. Robinson, "Practical methodology for modeling wireless routing protocols using OPNET modeler," in Proc. of 21st International Conference on Modeling and Simulation (MS 2010), 2010.

[21] (2013, January) The network simulator ns-2. http://www.isi.edu/nsnam/ns/.

[22] R. W. Nadir Shah, Depei Qian, "An efficient unstructured $p 2 p$ overlay for file sharing over manets using underlying reactive routing," Journal of Internet Technology, vol. 12 , no. 3, pp. 517-530, may 2011.

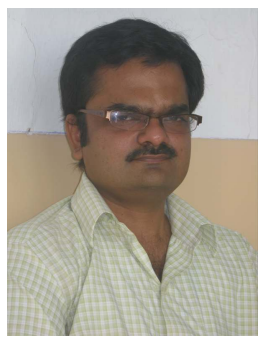

Vaibhav Godbole received Masters Degree in Electronics \& Telecommunication Engineering from Sardar Patel Institute of Technology, Mumbai and Bachelors Degree in Electrical Engineering from Sardar Patel College of Engineering, Mumbai, Diploma in Industrial Electronics from S.B.M. Polytechnic, Mumbai and Diploma in Mechanical Engineering from Govt. Polytechnic, Mumbai.He is currently working as a Assistant Professor at Fr. Conceicao Rodigues College Of Engineering, Mumbai. He is also a reviewer for IET Journal of Networks. His areas of interests are mobile \& wireless communications, evolutionary algorithms, genetic algorithms, new algorithms for mobile ad-hoc networks and wireless sensor networks. 